\title{
Colonización de hongos formadores de micorrizas arbusculares en raíces de pasto colosoana y angletón en cinco localidades del departamento de Sucre
}

\section{Colonization of forming arbuscular mycorrhizas fungi in roots colosoana and angleton grass in five localities of the department of Sucre}

\author{
PÉREZ C. CRISTO ${ }^{1}$ M.SC, PEROZA-CORONADO VICTOR ${ }^{2}$ M.Sc, \\ PÉREZ-CORDERO ALEXANDER ${ }^{3 *}$ Ph.D. \\ ${ }^{1}$ Fondo Nacional del Arroz, Fedearroz, Montería, Colombia. \\ 2Universidad de Sucre, Facultad de Ciencias Agropecuarias, Sincelejo, Colombia. \\ ${ }^{3}$ Universidad de Sucre, Grupo de Investigación Bioprospección Agropecuaria, Sincelejo, Colombia.
}

Keywords:

Livestock, pasture, rice, fungi.

\section{Abstract}

The present study was carried out during the second half of 2015 in livestock five localities of the Department of Sucre, Colombia, established with pastures colosoana (Bothriochloa pertusa (L)A. Camus) and angletón (Dichanthium aristatum Benth), with the objective of determining the percentage of colonization arbuscular mycorrhizal (AMF) in roots of these two species of pastures. Soils with presence of root samples were collected from five locations of the department of Sucre, from which it was determined the percentage of colonization in roots by HMA. The results of analysis of variance for percentage of colonization in roots of grasses colosoana and angletón ( $p$ value $=0.000$ ), respectively), finding the greatest percentage of colonization to the pasture colosoana $(36.9 \pm 79.09 \%)$ regarding the angletón grass $(33.3 \pm 72.72 \%)$ in five areas evaluated. The results show that both pastures is colonized by HMA.

\section{Resumen}

El presente estudio se realizó durante el segundo semestre de 2015 en fincas ganaderas de cinco localidades del departamento de Sucre, Colombia, establecidas con pastos colosoana (Bothriochloa pertusa (L) A. Camus) y angletón (Dichanthium aristatum, Benth), con el objetivo de determinar el porcentaje de colonización de hongos formadores de micorrizas arbusculares (HMA) en raíces de estas dos especies de pasturas. Las muestras de suelos con presencia de raíces fueron colectadas de cinco localidades del departamento de Sucre, a partir de la cuales se determinó el porcentaje de colonización en raíces por HMA. Los resultados de análisis de varianza para porcentaje de colonización en raíces de pastos colosoana y angletón ( $p$ value $=0,000$ ), respectivamente), encontrándose los mayores porcentaje de colonización para el pasto colosoana $(36.9 \pm 79.09 \%)$ con respecto al pasto angletón $(33.3 \pm 72.72 \%)$ en las cinco localidades evaluados. Los resultados obtenidos demuestra que ambas pasturas esta colonizadas por HMA. 


\section{Introducción}

Las pasturas colombianas en tierras del trópico bajo están constituidas por gramíneas de alto potencial productivo como Panicum maximum, Dichanthium aristatum, Brachiarias y algunas especies naturalizadas como Bothriochloa pertusa. Esta última ha colonizado en forma rápida diferentes tierras ganaderas, desplazando otras especies cultivadas (PIÑEROS et al., 2011). La principal actividad económica del departamento de Sucre es la ganadería doble propósito, en el cual el $84,9 \%$ de su territorio está dedicado exclusivamente al pastoreo de ganado donde la fuente exclusiva de alimento animal la constituye las leguminosas nativas y las gramíneas. Dentro de estas últimas, los pastos colosoana (Bothriochloa pertusa (L) A. Camus) y angletón (Dichanthium aristatum, Benth) son las especies predominantes en el departamento (AGUILERA, 2005).

Los hongos formadores de micorrizas arbusculares (HMA), son asociaciones ecológicamente mutualistas entre hongos del phyllum Glomeromycota (SHÜBLER et al., 2001) y la inmensa mayoría de la plantas cultivadas y silvestres (PÉREZ et al., 2011). Estos hongos son poco conocidos, pero de gran importancia para los ecosistemas terrestres. El termino micorriza hace referencia a la asociación simbiótica entre raíces de plantas y hongos, es llamada mutualista porque tanto los hongos como la planta hospedera se benefician. El hongo simbionte recibe carbohidratos de la planta ya que él es incapaz de realizar fotosíntesis y, a cambio, brinda a la planta varios beneficios reflejados en su crecimiento (JARAMILLO, 2011).

Estos microorganismos del suelo forman simbiosis con más del $95 \%$ de las plantas terrestres (MIRANSARI et al., 2009; STRULLUDERRIEN y STRULLU, 2007). Se ha demostrado que la asociación de estos hongos en distintas especies de gramíneas favorece la absorción, translocación y transferencia de los nutrientes y agua por la planta y que el principal nutriente implicado es el fósforo. Dichos hongos estimulan el crecimiento, desarrollo y nutrición de las plantas, especialmente en suelos de baja y moderada fertilidad. También se ha demostrado que estos hongos micorrízicos arbusculares mejoran la producción, la calidad nutricional e incrementan la tolerancia de las especies de pastos al pisoteo, debido al suministro de nutrientes hacia la planta, los cuales estimulan al rebrote continuo y a la recuperación rápida después de la desfoliación hecha por los animales herbívoros (AHN-HEUM et al., 2001).

Los hongos formadores de micorrizas arbusculares (HMA) son parte integral del sistema suelo. La rizósfera se compone del suelo cercano a las raíces de las plantas y es afectada por la actividad de ellas. La micorrizosfera es la zona del suelo afectada por la asociación micorrízica, la cual tiene dos componentes, la capa de suelo alrededor de las rices micorrizadas y la otra es el suelo cercano a las hifas del hongo micorrízica (HM) o micelio externo que compone la hifósfera o micósfera (JARAMILLO, 2011).

Sin embargo, hasta la fecha no se ha realizado un estudio significativo para determinar la colonización de HMA asociados a raíces de las especies de pasto colosoana y angletón bajo las condiciones edafoclimáticas del departamento de Sucre. Por tal razón esta investigación contribuirá al conocimiento de este tipo de asociaciones, para crear un punto de partida de futuras investigaciones a nivel de biodiversidad sobre estos microorganismos y su potencial uso como biofertilizantes en cultivos de importancia económica para la ganadería, planteando y desarrollando estrategias de uso y gestión racional de recursos disponibles del suelo, optimizando la productividad del sistema suelo - planta - animal.

Teniendo en cuenta la importancia de esta simbiosis natural y el valor de este proceso para los cultivos de pastos en la región, se planteó conocer la colonización en raíces por esto hongos en dos especies de pasturas de importancia para el departamento de Sucre.

\section{Materiales y métodos}

Sitio de estudio: Se realizó en el municipio de Corozal-Sucre (Colombia), ubicado en la región fisiográfica de Sabanas que constituye el declive general de los montes de María hacia la depresión del bajo Cauca y San Jorge; a los $8^{\circ} 55^{\prime \prime}$ y $9^{\circ} 19^{\prime \prime}$ de latitud Norte, y entre $75^{\circ} 25^{\prime \prime}$ y $74^{\circ} 42^{\prime \prime}$ al Este del meridiano de Greenwich; con temperatura promedio anual de $28^{\circ} \mathrm{C}$, pluviosidad de $1105 \mathrm{~mm}$ anuales y humedad relativa del $80 \%$, a una altura entre 174 a $200 \mathrm{msnm}$.

Recolección de muestras: Se seleccionaron al azar 100 fincas ganaderas distribuidas en cinco localidades del municipio de Corozal (Sucre) 
con pastura predominante de pasto colosoana $y$ angletón. En cada finca ganadera se realizó un muestreo aleatorio. En cada sitio se colectaron diez plantas completas (incluyendo raíces) de cada pastura. Las muestras fueron identificadas y almacenadas para el transporte al Laboratorio de Investigaciones Microbiológicas de la Universidad de Sucre y procesadas dentro de las 24 horas después de colectadas. De cada planta se separaron las raíces para para la determinación del porcentaje de colonización de HMA.

Determinación del porcentaje de colonización de HMA: El porcentaje de colonización de HMA en raíces de $B$. pertusa fue determinado mediante técnica de coloración de raíces. Las raíces coloreadas fueron colocadas sobre láminas cubiertas con laminillas, para su observación con objetivo 40X. Para estimar el porcentaje de colonización en raíces se contaron 100 campos ordenadamente por muestra. En cada muestra se observó la presencia de campos negativos (sin presencia de estructuras colonizantes) y positivos (con presencia de estructuras colonizantes). En los campos positivos se tuvo en cuenta el tipo de estructuras (arbúsculos, vesículas, hifas intra-radical, extra-radical, esporas, entre otras) presente en cada raíz (PÉREZ y VERTEL, 2010).

Análisis estadístico: Para interpretar la relación entre porcentaje de colonización en raíces por HMA en función a especies de pastura, se realizó un análisis de varianza de dos factores por grupo y prueba de Tukey para las diferentes interacciones.

\section{Resultados y discusión}

Las medias de los porcentajes de colonización de HMA en raíces de pasto colosoana y angletón por localidad (Tabla 1). El análisis de ANAVA, muestra diferencias significativas para porcentaje de colonización con relación a tipo de pasto y localidad. Los mayores porcentajes de colonización fue encontrado para el pasto colosoana $(79.09 \%)$ con respecto a pasto angletón (72,72\%). Para los pastos colosoana y angletón las mayores colonizaciones de HMA se presentaron en las fincas ganaderas localizadas en la localidad de Chapinero. Así mismo se observa que existen diferencias en cuanto a la colonización para ambas pasturas en las cinco localidades analizadas (Figura 1).

En trabajos realizados por PÉREZ et al., (2012), sobre colonización de HMA en pasto colosoana y angletón en el municipio de Corozal. Para el pasto
Tabla 1. Medias de porcentajes de colonización de hongos formadores de micorrizas arbusculares en pasto colosoana y angletón en cinco localidades del departamento de Sucre.

\begin{tabular}{lcc}
\hline \multicolumn{1}{c}{ Localidad } & \%colosoana & \%angletón \\
\hline Chapinero & 79.0909 & 72.7273 \\
Las Peñas & 58.6667 & 60.5833 \\
Hato Nuevo & 45.9091 & 46.8182 \\
Cantagallo & 42.8 & 38.5 \\
El Mamón & 37.6 & 33.3 \\
\hline
\end{tabular}

\% colonización de HMA en

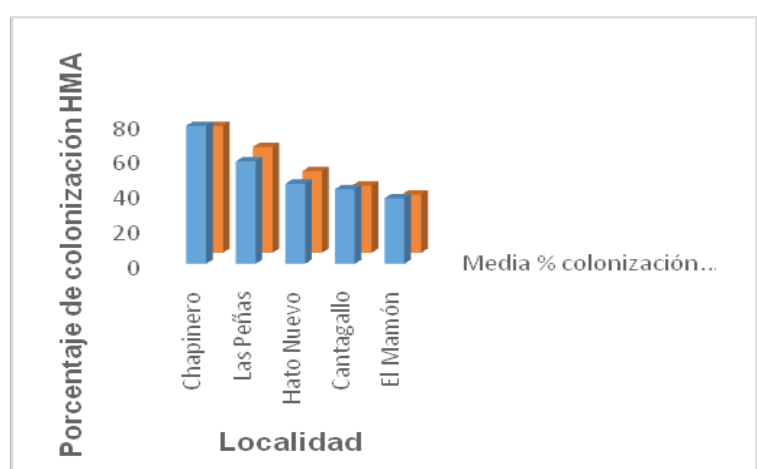

Figura 1. Medias de porcentajes de colonización de hongos formadores de micorrizas arbusculares en pasto colosoana y angletón en cinco localidades del departamento de Sucre

colosoana se reportaron los menores porcentajes de colonización de $45 \pm 76 \%$, mientras que para angletón se encontraron los mayores valores de colonización de $68 \pm 82 \%$. Con relación al presente estudio los resultados indican que los mayores porcentajes de colonización se encontraron para el pasto colosoana $(37,6 \pm$ $79,09 \%)$ y los menores para pasto angletón (33,3 $\pm 72,72 \%$ ). El establecimiento de la colonización de los HMA bajo condiciones de campo están determinado por diversos condiciones tales como: factores físico-químicas del suelo $\mathrm{pH}$, contenido de fósforo, temperatura, aireación, textura y contenido de materia orgánica), condiciones climáticas (intensidad y duración de la luz, temperaturas, humedad, épocas de lluvias y épocas secas) y por las prácticas agronómicas (preparación del terreno, aplicación de pesticidas y prácticas culturales) entre otros (PÉREZ y VERTEL, 2010).

Las estructuras de colonización encontradas en raíces de pasto colosoana y angletón correspondieron a: Hifas extra-radical, hifas intra- 
radicales, hifas extra-radical + esporas, esporas intra-radical y vesículas (Figura 2). Un total de 828 estructuras colonizantes fueron encontradas de las cuales 469 de reportan para pasto colosoana y 359 para pasto angletón. La estructura de mayor presencia en pasto colosoana correspondió a hifas intra-radical (163/469) y la menos abundante para vesículas (31/469). Así mismo se muestra que en pasto angletón predominó la presencia de hifas extra-radical (116/359) con respecto a las vesículas (17/359).

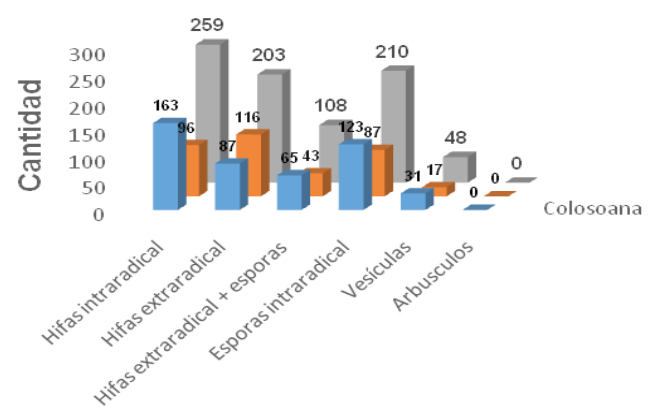

Tipo de estructuras colonizantes

Figura 2. Tipo de estructuras colonizantes observadas de HMA en raíces de pasto colosoana y angletón de diferentes fincas ganaderas del municipio de Corozal, departamento de Sucre, Colombia.

En la agricultura, el uso de HMA tiene un gran potencial biotecnológico debido a que facilitan la disponibilidad de nutrientes para las plantas. Por lo tanto, plantas micorrizadas poseen una ventaja importante con respecto a las plantas no micorrizadas (DA SILVA y CARDOSO, 2007). La importancia de los HMA en la agricultura radica en que por su extenso micelio extra radical, se forma un vínculo entre la planta y el suelo debido a que al darse la asociación planta-hongo, las plantas micorrizadas presentan ventajas en cuanto a la absorción de nutrientes de poca movilidad (como el P) con respecto a las plantas no micorrizadas, ya que en las primeras el micelio externo se extiende a una mayor distancia en el suelo que los pelos radicales de las plantas no micorrizadas (BLANCOF y SALAS, 1997).

Los hongos formadores de micorrizas arbusculares deben ser considerados, entonces, como parte de la diversidad biológica de los suelos y deben ser incluidos tanto en los inventarios como en los análisis de la biodiversidad a nivel de ecosistemas y agroecosistemas. La producción sostenible de pasturas en los trópicos es severamente limitada por la fragilidad de los suelos, siendo propensos a diversas formas de degradación. Hacer un mejor uso de los recursos biológicos en estos suelos puede contribuir a aumentar la sostenibilidad. Los hongos micorrízicos constituyen un recurso biológico importante en este sentido. Su contribución a la química biológica, y la calidad física del suelo ha sido reconocida, aunque aún quedan muchas preguntas de cómo utilizar de forma óptima estos hongos benéficos (PEREZ et al.; 2011).

Agradecimientos: Los autores expresan su agradecimiento trabajo laboratorio de investigaciones microbiológicas de la Universidad de Sucre por el apoyo en la ejecución de este.

\section{Referencias}

AGUILERA, M.M. 2005. Documento de trabajo sobre economía regional. Economía Regional: La Economía del Departamento de Sucre: Ganadería y Sector Público. Ediciones Banco Ganadero, Sincelejo, Colombia.

AHN-HEUM E.; GAIL, W.; HARTNETT, D. 2001. Effect of ungulate grazer on arbuscular mycorrhizal simbioses and fungal community structure in tall grass prairie. Mycología 93 (2):233 - 242.

BLANCOF, F.; SALAS, E. 1997. Micorrizas en la agricultura: contexto mundial e investigación realizada en Costa Rica. Agronomía Costarricense 21 (1):55-67.

DA SILVA, J.; CARDOSO, E. 2007. Micorriza arbuscular em cupuaçu e pupunha cultivados em sistema agroforestal e em monocultivo na Amazônia Central. Pesquisa Agropecuaria Brasileira 41 (5):819-825. 
JARAMILLO, R.I. 2011. La micorriza arbuscular (MA) centro de la rizósfera: comunidad microbiológica dinámica del suelo. Revista Contactos 81:17-23.

MIRANSARI, M.; BAHRAMI, H.A.; REJALI, F.; MALAKOUTI, M.J. 2009. Effects of soil compaction and arbuscular mycorrhiza on corn (Zea mays L.) nutrient uptake. Soil and Tillage Research 103 (2):282-290.

PEREZ, C. A.; ROJAS, S. J.; MONTES, V. D. 2011. Hongos formadores de micorrizas arbusculares: una alternativa biológica para la sostenibilidad de los agroecosistemas de praderas en el Caribe Colombiano. Rev. Colombiana cienc. Anim. 3 (2):366-385.

PÉREZ, C.A.; BOTERO, L.C.; CEPERO, G.M. 2012. Diversidad de micorrizas arbusculares en pasto colosoana (Bothriochloa pertusa (L) A. Camus de fincas ganaderas del municipio de Corozal-Sucre. Rev. MVZ Córdoba 17 (2):3024-3032.

PÉREZ, C. A.; VERTEL, M.M. 2010. Evaluación de la colonización de micorrizas arbusculares en pasto Bothriochloa pertusa (L) A. Camus. Rev. MVZ Córdoba. 15 (3):2165-2174.

PIÑEROS, V.R.; TOBAR, V.; MORA, D.J. 2011. Evaluación agronómica y zootécnica del pasto Colosoana (Bothriochloa pertusa) en el trópico seco del Tolima. Revista Colombiana de Ciencia Animal 4 (1):36-40.

SHÜßLER, A.; SCHWARZOTT, D.; WALKER, C. 2001. A new fungal phylum, the Glomeromycota: phylogeny and evolution. Mycological Research 105:1413-1421.

STRULLU-DERRIEN, CH.; STRULLU, D. G. 2007. Mycorrhization of fossil and living plants. C R Palevol $6(6-7): 483-494$. 